\title{
Development of a wheel robot and micro fling robot using for rescue scenarios
}

\author{
Nobuhiro Shimoi ${ }^{1, ~ *}$, Yoshihiro Takita ${ }^{2}$, Hirokazu Madokoro ${ }^{1}$ \\ ${ }^{1}$ Department of Machine Intelligence and Systems Engineering, Akita Prefectural University, Yurihoujo, 015-0055 Japan \\ ${ }^{2}$ Department of Computer Science, National Defence Academy, Yokosuka, 293-8686 Japan
}

\section{Email address:}

shimoi@akita-pu.ac.jp(N. Shimoi), takita@nda.ac.jp(Y. Takita)

\section{To cite this article:}

Nobuhiro Shimoi ,Yoshihiro Takita, Hirokazu Madokoro. Development of a Wheel Robot and Micro Fling Robot Using for Rescue Scenarios. American Journal of Remote Sensing. Vol. 1, No. 3, 2013, pp. 61-66. doi: 10.11648/j.ajrs.20130103.11

\begin{abstract}
In natural disaster, quick rescue is required. However, quick rescue is very difficult because victims are most likely to be left at the place where the rescue team are hard to find, such as under debris of broken house. The necessity for the rescue robot, which assists the rescue team by searching and finding victims of a disaster, is increasing. Nowadays many researchers and facilities are focus on studying these kinds of robots. However, the robot which can respond to various disaster-affected areas is not yet developed. The purpose of this study is to develop a rough terrain running rescue robot moving around the disaster-affected area of earthquake. In this paper, we first suggest mechanical structures and functions for the platform of the rescue robot. And then based on the suggestion, we develop a novel platform for rescue mobile robot RAT-1. Finally, experimental results show the effectiveness of the platform.
\end{abstract}

Keywords: Rescue Robot, Sensor of Rescue

\section{Introduction}

Unfortunately, on March 11, 2011, a massive earthquake and accompanying tsunami hit the Tohoku region of eastern Japan, claiming many lives. The number of collapsed houses totaled more than 350,000 , and over 20,000 persons were confirmed as either missing or dead. The Fukushima Daiichi Nuclear Power Plants also sustained extensive damage in the disaster, which resulted in total loss of power, and meltdown accidents during which radioactive materials were released. In the final analysis, three buildings housing nuclear reactors were seriously damaged because of hydrogen explosions and, in one building, the nuclear reactions were also out of control. The condition of the reactors was recently stabilized as their cores are currently being cooled by water[1]-[3]

After an earthquake, it is very difficult to locate survivors who are confined in closed spaces such as underground passages and tunnels, where rescuers cannot get closer because of rubble. Rescue robots are used in such situations. However, complex systems, sensors, sophisticated control techniques, etc., are required for the robots to climb up and down the rubble while searching[4]. To resolve such problems, this studied considered a method for searching for survivors and grasping the damage situation using an ultra small infrared camera, biosensor, etc., mounted on a micro flying robot that flies in the space between the rubble and the ceiling.

Original devices and systems were adopted for the design and manufacture of the micro flying robot and control system used in rescue missions[5]-[7].

As one means, such a problems exploration using mobile robot technology was crucial for this and subsequent missions. We would like to proposal using the Rescue robot RAT-1 and fling robot MOSQUIO-1 for developing tracked robots to assist rescue crews in search and rescue missions in dangerous environments, particularly in underground malls[8]-[11].

Fig. 1 shows the remote controlled mobile robot, RAT-1.

It is very compact and convenient. This paper proposes an wheel with wheels and arm mechanisms which have the ability to climb over obstacles such as stairs. Each mechanism consists of an arm with two wheels mounted on either side. In order words, this is a system which utilizes a planetary gear without an inner gear. Two pairs of mechanisms are combined into one package: one is installed in front and one on the rear of the robot body and their directional angles are controlled by the servo motors.

And then Fig. 2 shows the autonomous control fling robot MOSQUITO-1.This robot is task outside sensor and 
reconnaissance flight for operation of rescue missions.

The amplifier, driving power supply, receiver, control substrate, etc., incorporated into the machine body were miniaturized as much as possible. Flight control has two modes: manual control, which uses a proportional control system; and sub-autonomous flight, which uses a mechanism for active shifting of the center of gravity with PID (Proportional Integral Differential) control System by PIC micro computer[12][13][14].

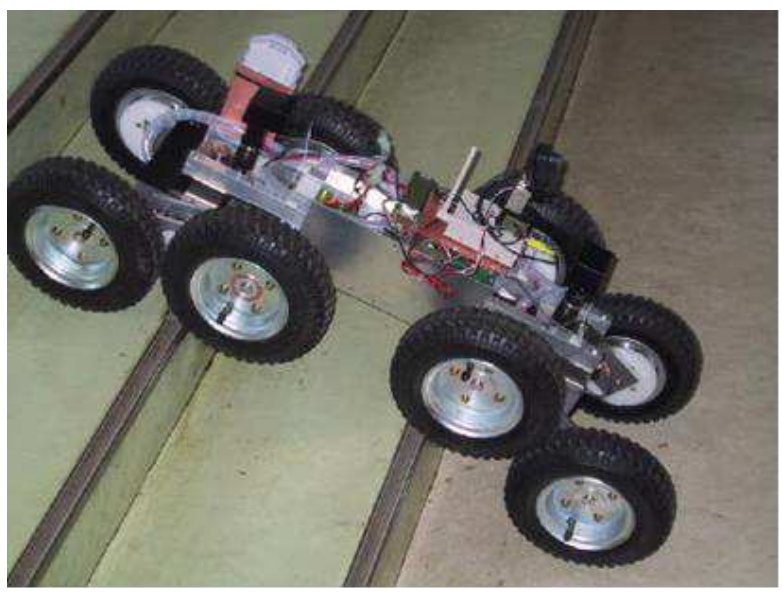

Figure 1. Remote controlled mobile robot RAT-1.

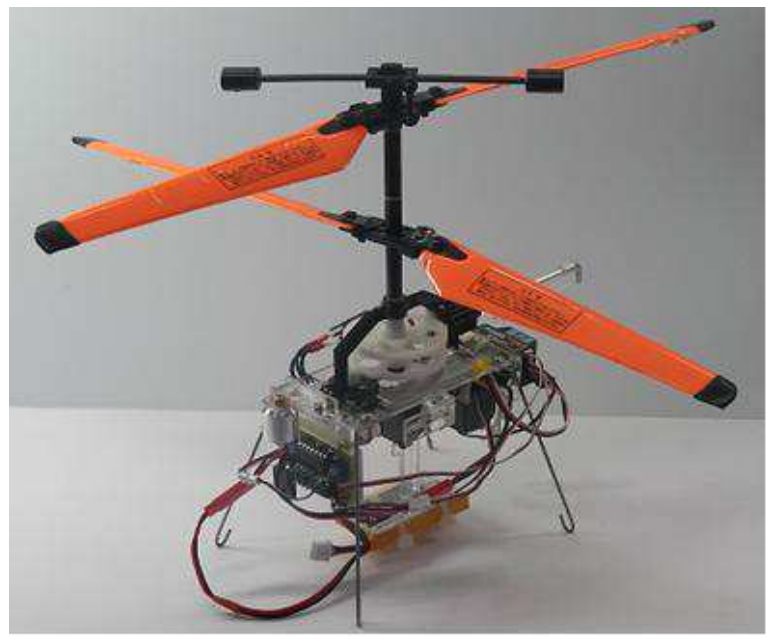

Figure 2. Autonomous control fling robot MOSQUITO-1.

\section{Rescue Robot RAT-1}

\subsection{Outline of the Robot}

The structure of the self standing mobile robot "RAT-1 Octal Wheel" system used for trial purposes in this research is shown in Fig.3. Here the diameter of tire D is $0.205 \mathrm{~m}$, the wheel distance $\mathrm{L}$ is $0.216 \mathrm{~m}$, the wheel base is $0.5 \mathrm{~m}$, and the tread is $0.31 \mathrm{~m}$. The RAT- 1 is equipped with a battery, a controller, a video camera and its transmitter, and a serial wireless transceiver in its body. Based on the construction of this system, the handler is able to operate the robot while monitoring video signals received with the remote control terminal.
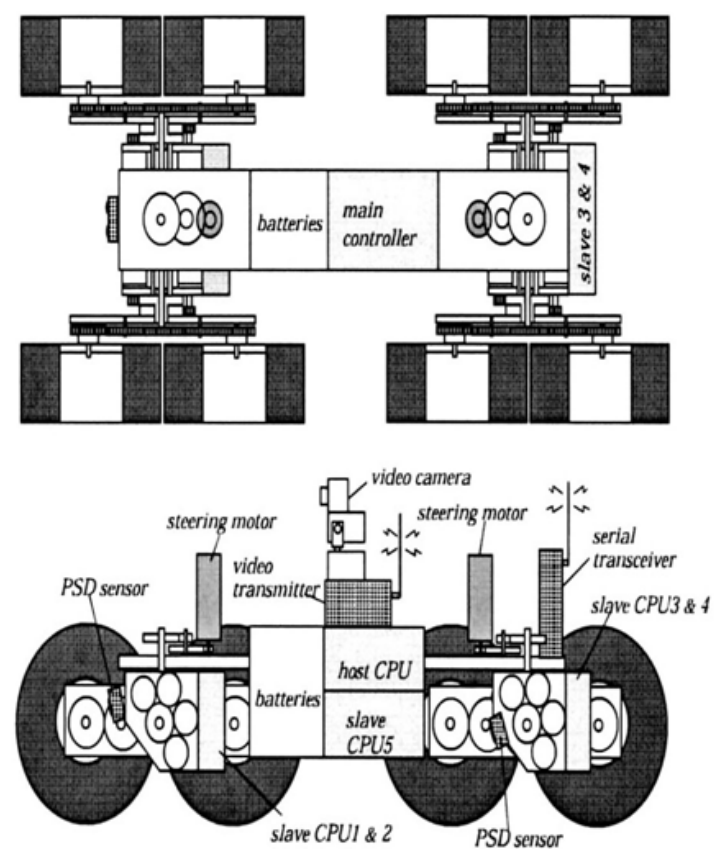

Figure 3. Outline of Robots wheels .

\subsection{Mechanism of Robots Wheel}

Fig.4 shows the crawler mechanism of the wheel-arm robot RAT-1 developed in this work. The shafts of tires A and B are fixed at each side of the arm. Each tire is driven by a servo motor rotating in the same direction as the contraction of the planetary gear system. Arm $\mathrm{C}$ is driven by another servo motor without interference from the tire drive mechanism. Fig.5 shows the procedure used for climbing over an obstacle whose height is equal to the diameter of the robot wheels. When tire A comes into contact with an obstacle, the controller will enable arm $\mathrm{C}$ to turn 180 degrees in a clockwise direction with constant rotational speed. When tire $\mathrm{B}$ contacts the obstacle, the robot is able to climb up the obstacle without resting. This motion is dependent on distance $\mathrm{L}$, the coefficient of static friction with the obstacle, and the tire's diameter D.

\subsection{Construction of the Control System}

It is necessary to develop a controller which has the capacity to monitor ten DC motors utilizing rotary encoders. The design method is different from the case of using only one CPU. In this case, it is difficult to sufficiently control each motor while processing the remote control signals. Therefore, we adopt a distributed control method for multiple CPUs in this system. The advantage of this method is that each program is simplified and executed at high speed, though communication between the CPUs then becomes a problem.

Fig. 6 and 7 shows the outline of the control system. One slave CPU controls two DC motors. A host CPU has concentrated control of the slave CPUs. The H8S/2250 CPU was selected for this system because it is composed of two channels of up/down counters for the rotary encoders and the PD control program for the two DC motors, show 
in Fig. 8, can be executed at sampling intervals of about $30 \mu \mathrm{s}$.

\subsection{Obstacle Detection Sensor}

Upon encountering an obstacle the robot is able to measure the distance between its tire and the obstruction and is then able to move its arm at the appropriate time. By equipping it with this sensor the robot is able to sense the obstacle and is then able to surmount it. This robot is equipped with the PSD distance sensor GP2D12 which determines the distance of the obstacle using an analog signal. The detectable range of this sensor is large arias, from 0.1 to $0.8 \mathrm{~m}$, and the measurement accuracy is influenced by the reflectivity of the object. The sensor is installed in the casing of the drive motors in the front and the rear of the robot, and is angled about 20 degrees downward to compensate for the changing posture of the body.

\subsection{Remote Control}

This robot is designed for rescue underground passages and tunnels. And there needs to function in an environment of uneven terrain. It works more realistic to construct a simple control system because autonomous movement and work are difficult to archive using present technology.

Therefore, in order to show the operator an accurate image for remote control, a CCD camera is installed on the pan head that turns automatically up and down and right and left with that signal then being transmitted with the video transmitter.
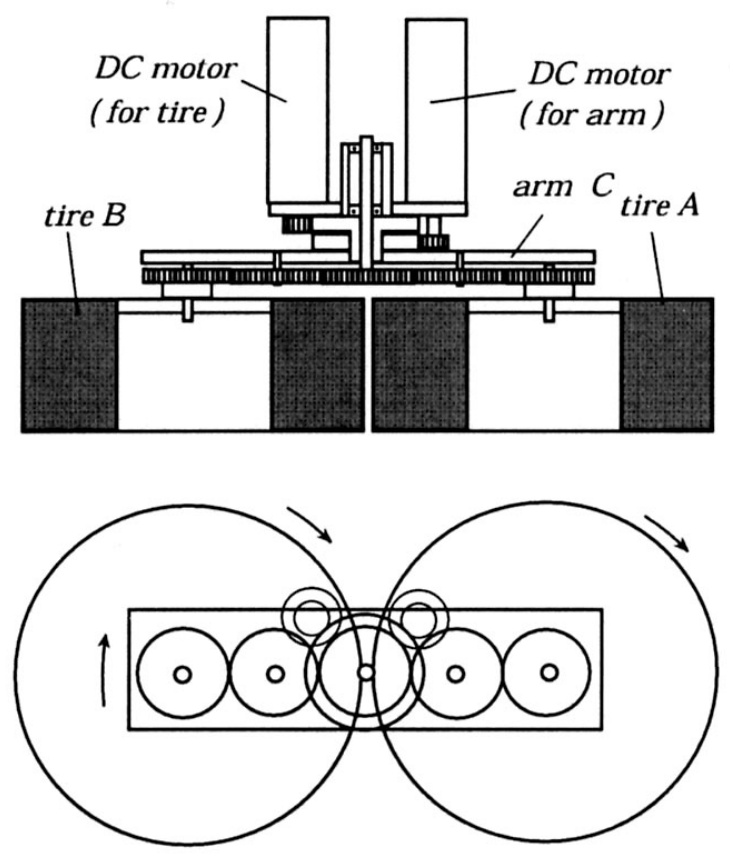

Figure 4. Quarter mechanism of a RAT-1

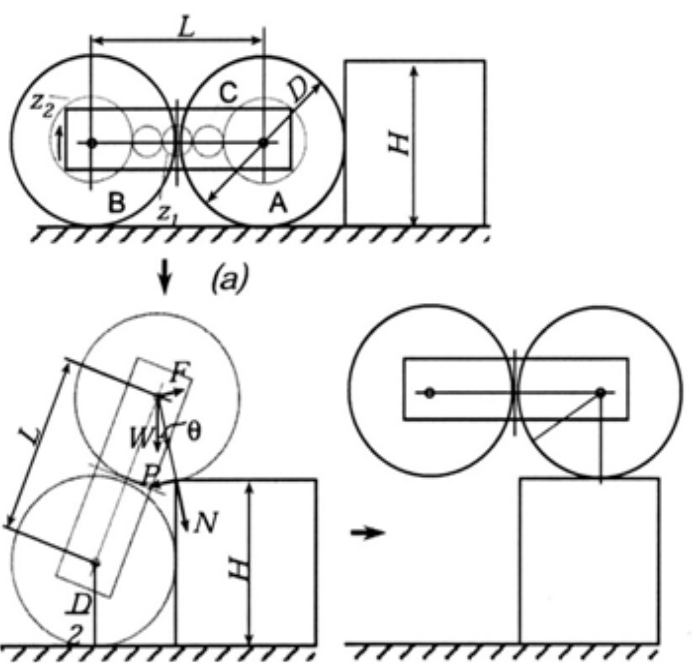

(b)

Figure 5. Schematic concept of climbing motion over an obstacle.

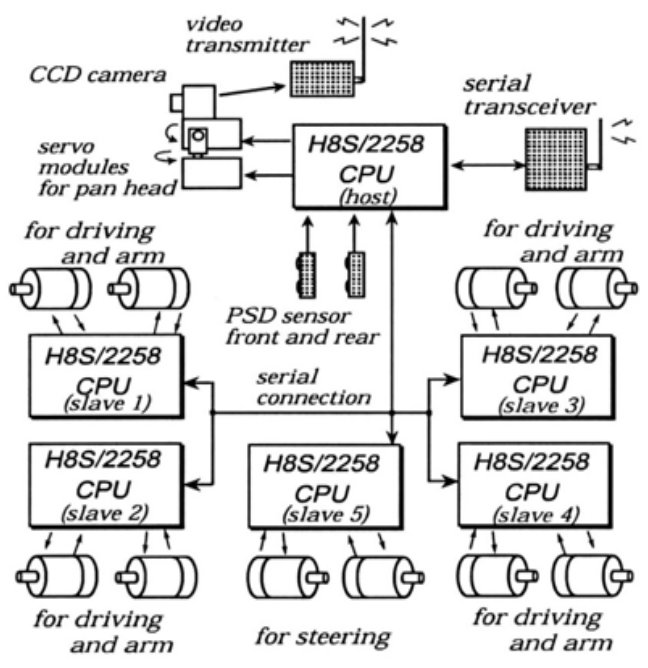

Figure 6. Outline of RAT-1 mechanism.

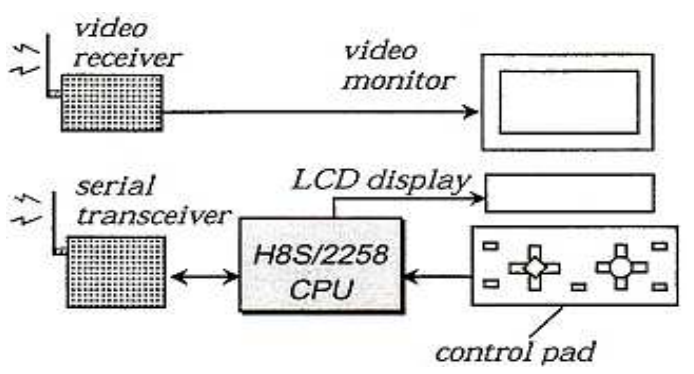

Figure 7. Remote control terminal

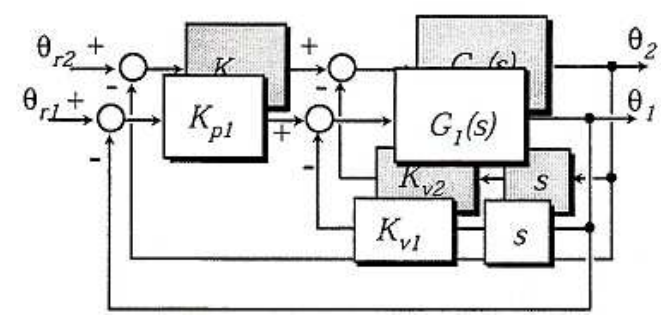

Figure 8. PD control realized by a controller 


\section{Fling Robot of Reconnaissance}

The design, manufacture, and assembly of the components were conducted in each process; after the operation was checked, the flying robot was manufactured, and the test flight was implemented. The outline of the complete micro flying robot is given in Table.1.

Table 1. Preface of flying machine

\begin{tabular}{ll}
\hline Items & Size \\
\hline Weight & $250 \mathrm{~g}$ \\
Lift of max & $3.528 \mathrm{~N}$ \\
Total length & $225 \mathrm{~mm}$ \\
Height & $240 \mathrm{~mm}$ \\
Width & $82 \mathrm{~mm}$ \\
Diameter (wings) & $352 \mathrm{~mm}$ \\
\hline
\end{tabular}

Flight stability was compared for two control modes: a combination of a mechanism for active shifting of the center of gravity, which is important for stable flight control when designing the machine body, and PID control; and a control mode that uses the mechanism for active shifting of the center of gravity only.

\subsection{Center of Gravity Positioning Mechanisms}

Fig.9 shows the details of a device that enables fine adjustment of the center of gravity from side to side and back to front by an adjustable weight attached to the front of the micro flying robot for stable takeoff of the machine.

Fig. 10 shows the details of the mechanism for active shifting of the center of gravity with a servo that automatically recognizes and controls the center of gravity of the machine body. This mechanism controls the battery power source as the weight to adjust the center of gravity when immediate stabilization of the machine is needed.

\subsection{Correction of Rotation}

The contra-rotating mechanism controls the rotation of the machine by rotating the upper and lower rotors in reverse directions at the same speed. However, if a stabilizer is connected to the upper rotor, a moment of inertia is generated, and a reaction moment to cancel it is required. We developed a method to avoid rotation by attaching a weight with the same moment of inertia as the stabilizer to the lower rotor based on our experimental results.

\subsection{Manual Control}

The proportional control system 4EX-Epset (Futaba) was used for manual control of the micro flying robot. The design was made so that it would be possible to control the three servo motors that shift the center of gravity and drive the steering mechanism and the brushless motor that drives the main rotor with the remote controller.

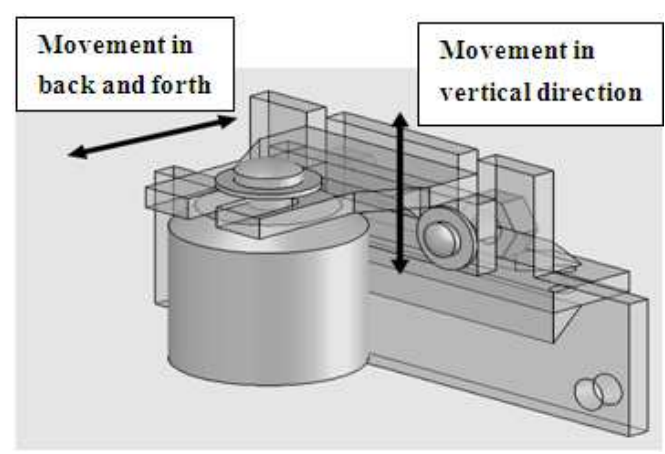

Figure 9. Center of gravity a front adjustment device

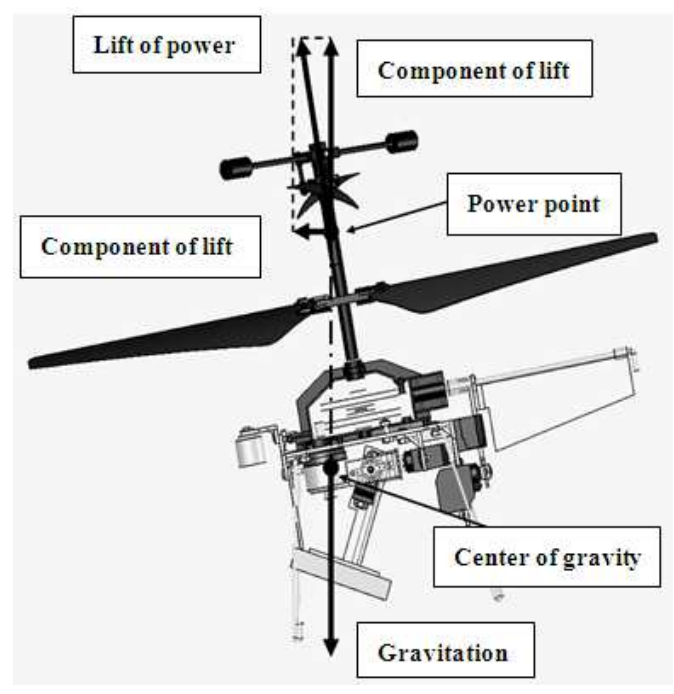

Figure 10. Center of gravity an active adjustment device

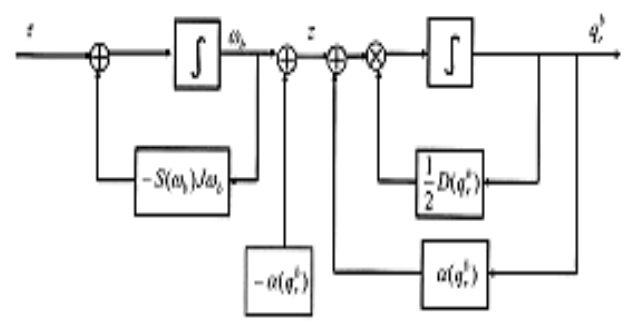

Figure 11. Block diagram of flight attitude control

\subsection{Auxiliary Control Function}

Feedback control with an acceleration sensor was implemented to improve stability when the machine was hovering or flying in a straight line. To implement the above control, the stability was tested when the robot was hovering by the control of center of gravity by the mechanism for active shifting of the center of gravity and PID control by PIC.

\section{Experiment of Result}

\subsection{Rat-1 Control of Moving at Stairs}

Fig. 12 show the way of going up and going down stairs based on a mechanical constraint. Here, the height and the depth of the stair is $0.18 \mathrm{~m}$ respectively. 


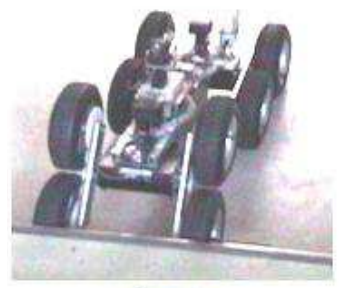

Start

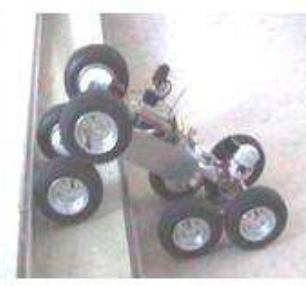

(b)

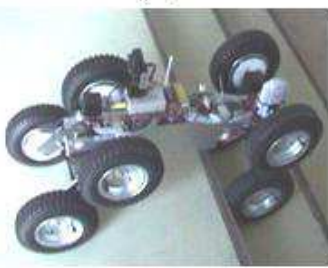

(d)

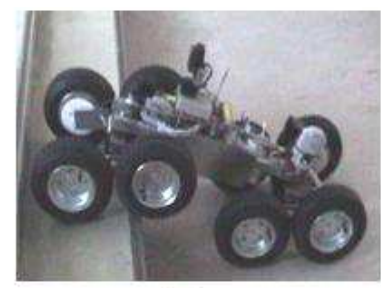

(a)

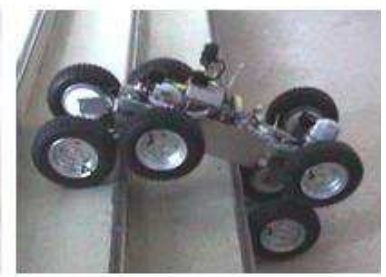

(c)

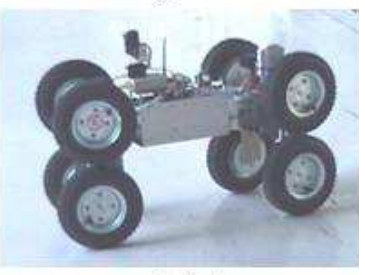

Finish
Figure 12. Realized climbing up motion of RAT-1

The (a) front wheel arm starts to rotate while the rear wheel is driven at a constant speed without the arm control. In order to prevent pushing against the step by the front tires, the reverse control is executed. In the (b) wheels stop reversing when the tire comes in contact with the next step and climbing force is obtained. Though it is difficult for the main body of the robot to distinguish the state of (b) and (d), the main value of both is used here.

The Fig.12 (c) shows that the initiation of the turn is obtained by measuring the distance to step obtained from the PSD sensor located at the front wheel, causing the front arm to rotate as well, as shown in (a). Control of the rear arm can similarly be executed using the rear PSD sensor.

Table 2. Calculated value ( $\mathrm{mm}$ )

\begin{tabular}{ccc}
\hline & Average & Standard deviation \\
(a) & 230 & 185 \\
b) & 120 & 95 \\
\hline
\end{tabular}

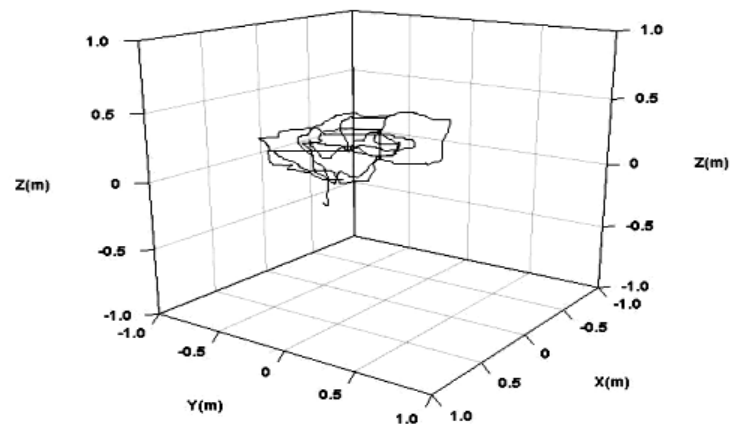

(a) Free control of flight a locus

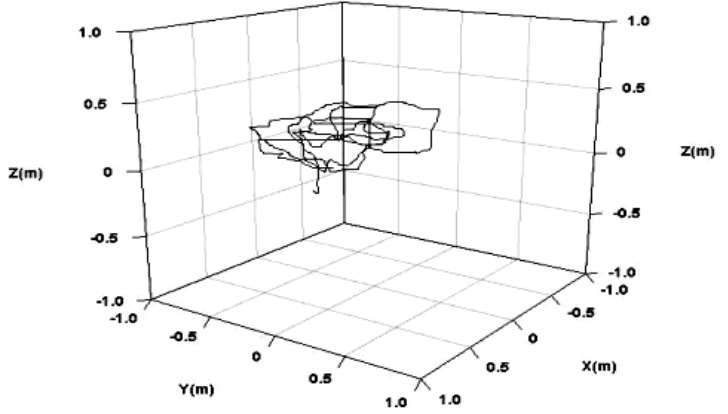

(b) Without PID control of flight a locus

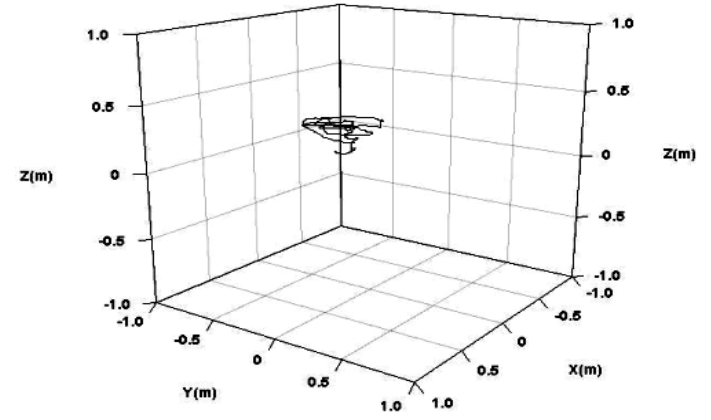

(c) With PID control and active machinery of flight a locus

Figure 13. Realized fling motion test of Mosqito-1

\subsection{Mosquito-1 Control of Flight}

Fig.13 shows the measurement results for the three control modes. Table 2 presents the average movement distance and standard deviation of each mode. During the takeoff/landing test, the flying machine rotated more than expected. This was because, though the moments of inertia of the upper and lower rotors were balanced by the contra-rotating mechanism of the main rotor, the moment of inertia generated at the stabilizer was not taken into consideration. This was corrected by decreasing the rotation speed of the rotor so that the steering mechanism for controlling this rotation phenomenon could operate stably.

Without control in the (a), the machine was very unstable; the balance in the vertical and horizontal directions was lost, and the radius of the average distance of movement of the flight from the center position was about $400 \mathrm{~mm}$ or more.

When the machine was controlled with the mechanism for active shifting of the center of gravity, the radius of the average distance of movement was reduced to about 230 $\mathrm{mm}$, and it was more stable in the (c). When the machine was controlled with the combination of the mechanism for active shifting of the center of gravity and PID control, the radius of the average distance of movement was about 120 $\mathrm{mm}$, and the robot could fly stably in the (d). Based on the above results, implementation of complex control was proved to reduce the deviation and variation in movement, and the effectiveness of the auxiliary control function combining the mechanism of active shifting of the center of gravity and PID control was confirmed. However, since there were many variables in this test, the test evaluation 
method, measuring technique, etc., should be improved for more accurate evaluation of the performance. Using PID was considered in this test, but comparison and evaluation with other methods, such as target control using a camera, are also important.

\section{Conclusion}

For each experimental device to be introduced in our Rescue robot system, various parameter values will require adaptation. With this in mind, simulation models will be generated to approximate the behavior of our robot with experimental devices. The simulation model of the eight-wheeled driving robot RAT-1 requires improvements in its initial status and maximum stride.

And then, Basic research on the flying robot Mosquto-1 made checking its performance using numeric data possible, and basic data on control devices and methods were acquired. This device should be effective as an auxiliary machine for disaster rescue systems, and further research to compare the control methods using a simulator and actual machine in terms of the search abilities of biosensors, etc., is important for rescue robot.

This work serves as a stepping-stone for the development of rescue robot technology for Rescue Scenarios purposes.

\section{References}

[1] Hiroaki Nakanishi, Mai Bando, Koichi Inoue, Akira Sato, "Research and development for intelligent aero robot for disaster prevention", International Rescue System Institute Quarterly Report, vol.1, pp14-15, 2005.

[2] Ichiro Fukuda, Yasushi Hada, Naoji Shiroma, Hajime Asama, Kuniaki Kawabata, Fumitoshi Matsuno, "The generation of 3-D terrain maps including information on moving object for disaster victim search", $6{ }^{\text {th }}$ Proceedings of the system Integration Division, (S12005), p p751-752, 2005.

[3] Koichi Osuga, "To construct the culture to think about rescue", $44^{\text {th }}$ Proceedings of the Institute of Systems, Control and Information Engineers, pp267-268, 2000.

[4] Hiroshi Otake, Ken Iimura, Kazuo Tanaka, "Fuzzy control of micro RC helicopter with coaxial counter-rotating blades (intelligent sensing and control)", Journal of the Japan Society for Fuzzy Theory and Intelligent Informatics, vol.21, no.1, pp10-102.

[5] Nobuhiro Shimoi, YoshihiroTakita, "Remote mine sensing technology using a Mobile Wheeled Robot RAT-1", Proceedings of the International Conference on Control Automation and Systems. ICCAS 2010, pp TE06-4(5)(2010.10)

[6] N. Shimoi, S. Koga, K. Itoh, et. Al, "Vibration reduction study with air-cushion”, CICE, Vol. 33, No. 5, pp. 337-343 (1997).

[7] Nobuhiro Shimoi, "The Subject NGO of the technology for mine detecting", JSME 99, D\&D, Vol. B99-7, pp. 277-280. 1999.7

[8] Nobuhiro Shimoi, "Development and examination for vertical take off and landing of the micro autonomy fling robot", $47^{\text {th }}$ Proceedings of the Touhoku erias.Japan society of Mechanical Engineers.pp108-109 , 2011.9.21

[9] Y.Takita, N. Shimoi," Development of Wheeled Mobil Robot Octal Wheel Realized Climbing Up and Down Stairs", Proceedings of 2004 IEEE/RSJ International Conference on Intelligent Robots and Systems, pp2440-2445(2004)

[10] Kazuhiro Igarashi, Kensaku Hazawa, Shin Jinok, Daigo Fujiwara, et al, "Autonomous small unmanned helicopter height and automatic taking-off and landing control", The $8^{\text {th }}$ Dynamics and Design Conference, pp91-92, 2003.

[11] Richard P. Olenick and Tom M. Apostol and David L. Goodstein," The Mechanical Universe", Introduction to Mechanics and Heat. Cambridge University Press, New York, 1985, pp 82.

[12] Ken Shoemake. Animating Rotations with Quaternion Curves, " In Brian A. Barsky, editor, Computer Graphics(SIGGRAPH '85 Proceedings)", volume 19, pp 245-254, July 1985.

[13] N. Shimoi, H. Madokpro, "Flight stabilization of micro fling robot for rescue sennarios", ICGST-ARAS Journa 1 ,vol 12,issue1,pp11-15,2012.1

[14] N. Shimoi, C. H. Cuadra, H. Madokoro, and M. Saijo, "Simple Smart Piezoelectric Bolt Sensor for Structural Monitoring of Bridges", International Journal of Instru mentation Science, vol.1, no.5, pp.78-83, Dec. 2012. 